\title{
IgG4-related Sclerosing Diseases Involving Abdominal Organs; Radiologic Findings and Differential Diagnosis
}

Young Hwan Lee MD, Seri Kang MD, Youe Ree Kim MD, Hye Ji Rue MD, Kwon-Ha Yoon MD Department of Radiology, Wonkwang University Hospital, Iksan, Korea

\section{Background}

Immunoglobulin G4 (IgG4)-related disease is a recently proposed clinical-pathologic entity characterized by fibroinflammatory lesions rich in IgG4-positive plasma cells and, often but not always, elevated serum IgG4 concentrations.

Although definitive diagnosis requires histopathologic analysis, imaging plays an important role in demonstrating infiltration and enlargement of involved organs. Because IgG4-related disease usually shows a marked response to corticosteroid therapy, radiologists should be familiar with its clinical and imaging manifestations to avoid a delayed diagnosis and unnecessary surgical interventions.

\section{Learning objectives}

1. To illustrate imaging findings of IgG4-related sclerosing diseases affecting various abdominal organs including Liver, biliary tract, pancreas, retroperitoneum, kidneys, lymph nodes.

2. To inform the imaging features of IgG4-related diseases of abdomen which can be helpful to differentiate from many of tumorous or other inflammatory abdominal diseases.

\section{Imaging Findings/Procedure Details}

IgG4-related Diseases of Abdomen and their Radiologic Mimics \begin{tabular}{l|l|l} 
Organs & IgG4-related Diseases & Mimics
\end{tabular}

Pancreas $\underline{\text { AIP }}$

Liver

Bilary tree

Kidney

Tubulointerstitial nephritis, Membranous

glomerulonephritis,

Inflammatory

Small bowel, $\underline{\operatorname{Ig} G 4-\text { related sclerosing }}$ mesentery

Retroperiton -eum

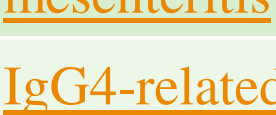

Lymph nodes

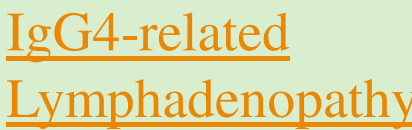

Focal form AIP - pancreatic malignancy

Diffuse form AIP - pancreatitis Malignant hepatic tumors

Primary sclerosing cholangitis, cholangiocarcinoma

Bilateral - lymphoma, metastases,

Unilateral- RCC

transitional cell carcinoma

Wedge shape - infarct, pyelonephritis

Lymphoma, carcinoid tumor, carcinomatosis

Lymphoma, large-vessel

vasculitis, syphilis,

sarcoidosis-induced arteritis

Lymphoma, multicentric

Castleman disease

disseminated malignancy

\section{IgG4-related Autoimmune Pancreatitis}

Specific findings: Capsulelike rim or halo of low attenuation surrounding pancreas, presumed to represent a fluid collection, a phlegmon, or fibrosis.

Imaging- Hypoechoic at US, hypoattenating at CT, midly hyperintense at T2-weighted

MR imaging with distinctive delayed contrast enhancement.

Diffuse diseases; a uniformly enlarged pancreas with sharp margin and loss of lobular contours, resulting in a featureless sausage like appearance

Focal disease; focal enlargement of pancreas, resulting in a mass like appearance

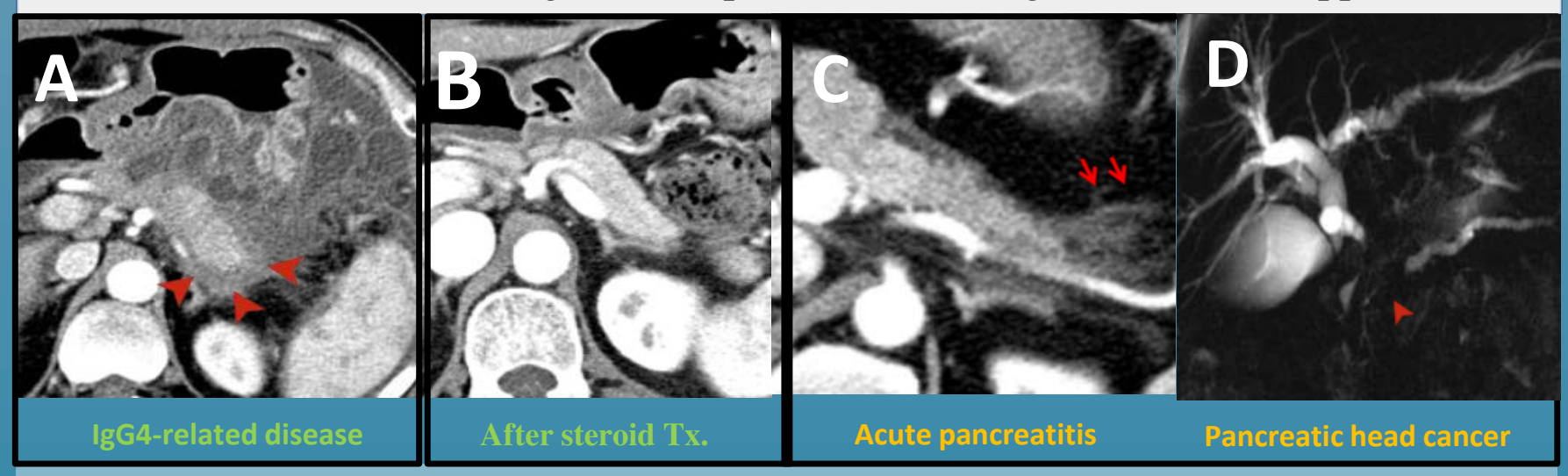

Fig.1 IgG4-related disease in a 50-year-old man (A,B). Acute pancreatitis in a 42-year-old woman (C). Pancreatic head cancer in a 69-year-old woman (D).

Axial contrast-enhanced (A) CT show swelling of the pancreatic tail with peripancreatic hypoattenuating halo(arrowhead). This patient shows marked elevation of IgG4 level with 883g/L. Follow-up CT image(B) obtained after steroid administration shows a marked response to treatment, with resolution of the hypoattenuating peripancreatic halo. Another patient with acute pancreattiis reveals peripancreatic fat st on enhanced CT ( $C$, arrows). Another patient with pancreatic head cancer reveals abrupt obstruction in the main pancreatic duct (arrowhead) with dilatation of upstream pancreatic duct and bile duct(double duct sign) on MRCP (D).

\section{IgG4-related Diseases of the Liver}

Two types of IgG4-related inflammatory pseudotumors;

Fibrohistiocytic, lymphoplasmacytic : more strong association

hypointense on T1-WI, hyperintense on T2-WI images with variable enhancement. Malignant hepatic tumor; rarely delayed enhancement on CT, need biopsy

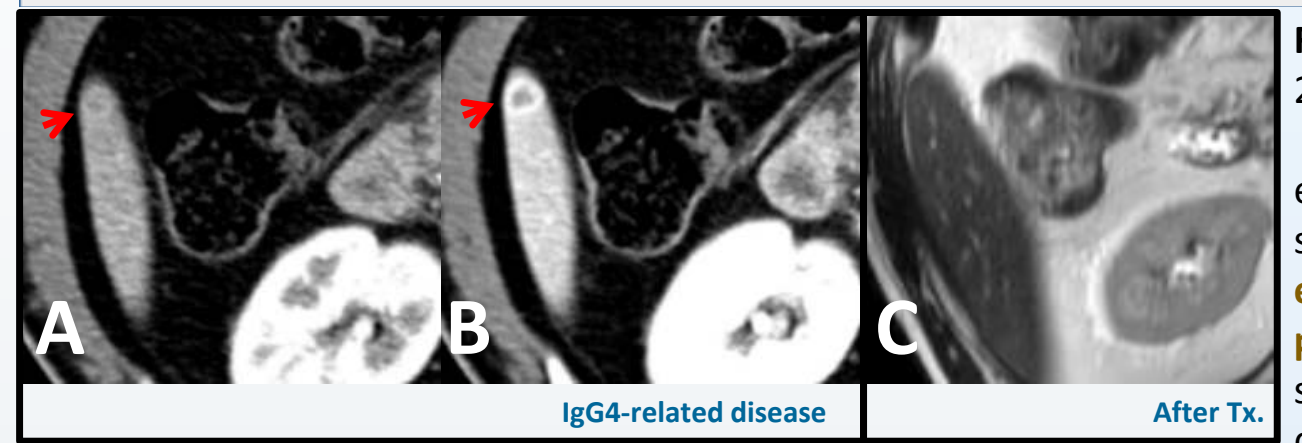

Fig.2 IgG4-related disease in 29-year-old man. Axial CT reveals a arterial enhancing nodule in the liver segment $6(A)$ with further enhancement in the delayed phase (B). 6 Months after

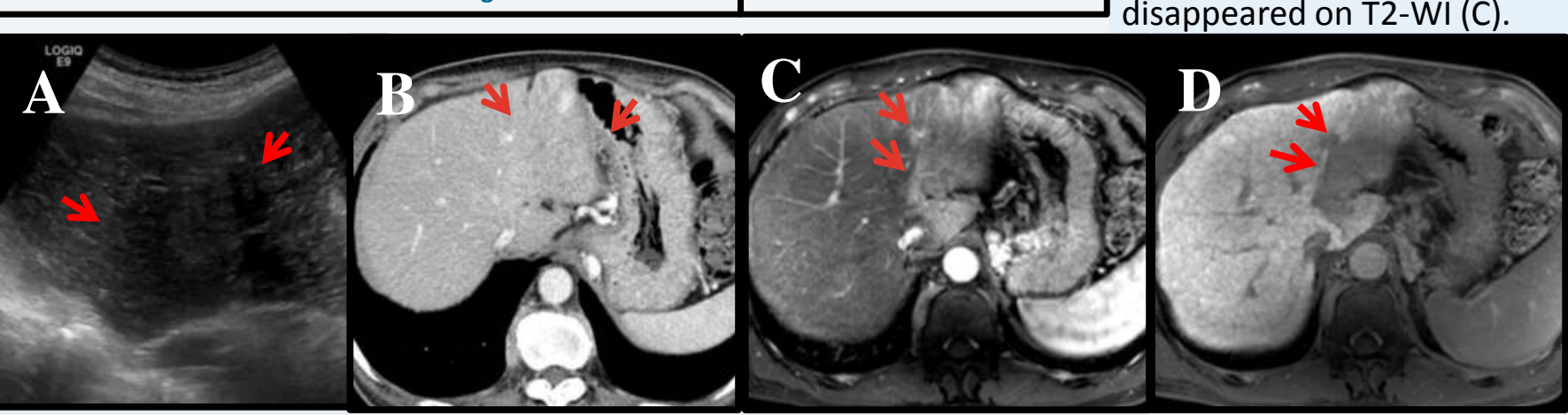

Fig.3 IgG4-related hepatopathy in a 54-year-old man.

Transabdominal ultrasound (A), contrast enhanced CT (B), axial fat saturated T2-WI (C) and Gd-EOB enhanced hepatobiliary phase image (D) show ill defined infiltrative hypoechoic hepatic mass with mild arterial enhancing and delayed washout, EOB defect, T2 hyperintensity, diffusion restriction (not shown) at lateral segment of left hepatic lobe and atrophic change of left portal vein is noted.

\section{IgG4-related Sclerosing Cholangitis}

Most common involved segment - the intrapancreatic portion of the

CBD. Focal or diffuse bile duct wall thickening with stenosis and upstream dilatation.

A circular and symmetric rind of tissue encasing the bile duct wall with relatively smooth margins and homogeneous enhancement in the delayed phase of CT and MRI.

Gallbladder involvement - diffuse wall thickening with decreased echogenicity at US and low signal intensity at T2-weighted MRI

with late enhancement.

Differential diagnosis are primary sclerosing cholangitis,

cholangioCa

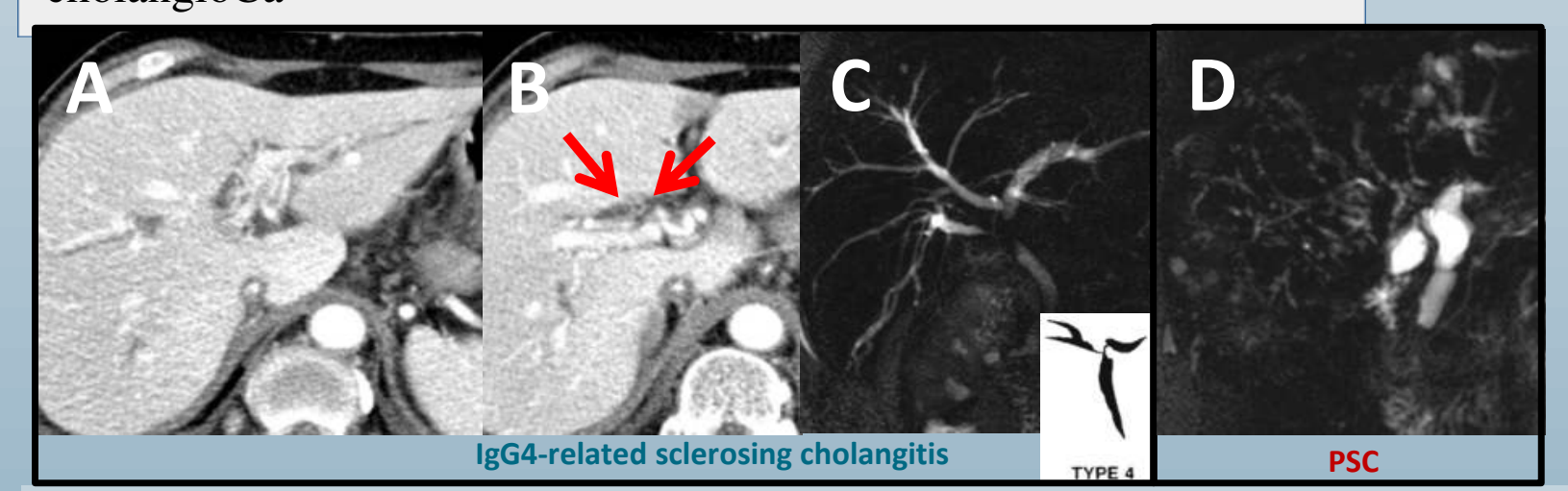

Fig.4 IgG4-related sclerosing cholangitis in a 62-year-old man (A, B, C). Primary sclerosing cholangitis (PSC) in 66-year-old woman (D). Axial contrast-enhanced CT image demonstrate concentric mild enhancing wall thickening of the common hepatic duct (B, arrows) with prestenotic intrahepatic bile duct dilatation(A). MRCP image also show stricture of extrahepatic bile duct and right intrahepatic bile duct at hepatic hilum(C). Another patient with primary sclerosing cholangitis reveals multifocal and short strictures of both intrahepatic bile duct with beaded appearance (D).

\section{IgG4-related Renal Involvement}

\section{Five patterns of diseases;}

1) bilateral round or wedge-shapedp

2) bilateral nodules in renal sinuses

3) a rim of soft tissue around the kidney

4) diffuse patchy involvement

5) diffuse wall thickening of renal pelvis

CT - hypoattenuating during the arterial phase, becoming

soattenuating relative to the surrounding parenchyma

during late phases.

MRI- low signal intensity on both T1- and T2-WI, with

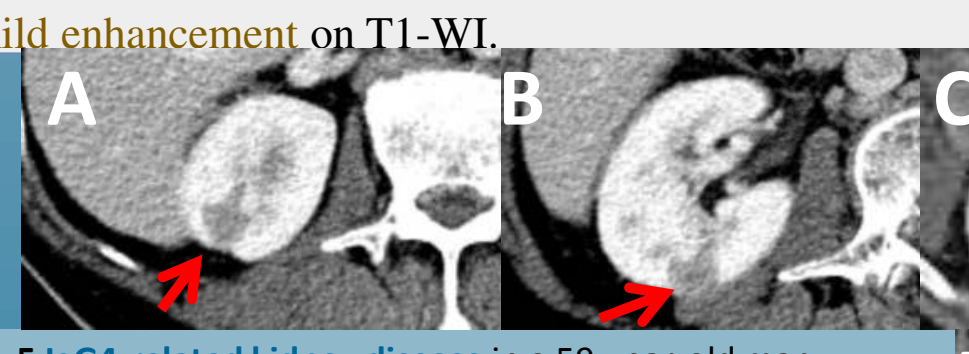

Fig.5 IgG4-related kidney disease in a 59-year-old man. Axial contrast-enhanced $\mathrm{CT}$ images show multiple enhan solid masses (arrow) in the right kidney (A, B) and also demonstrates ill defined enhancing soft tissue lesion along the eft renal pelvis (C, arrowhead). This pationt elevation of serum IgG4 level with plasma cell in biopsy. Two months after steroid therapy, soft tissue lesion along the left renal pelvis is almost not seen (D).

\section{Conclusion}

IgG4-related disease is a unique and distinct systemic disease that is frequently misdiagnosed as neoplastic and other inflammatory processes.

The recognition of the typical imaging patterns in each abdominal organ can raise the suspicion of the disease, and the combination of radiologic, serologic, and histologic findings can lead to correct diagnosis, ensuring effective treatment. 